\title{
Corela
}

Cognition, représentation, langage

$13-2 \mid 2015$

Vol. $13, n^{\circ} 2$

\section{The boxer sleeps. Un générique particulier : le générique intitulatif}

\section{Viviane Arigne}

\section{CpenEdition}

\section{Journals}

Édition électronique

URL : http://journals.openedition.org/corela/4754

DOI : $10.4000 /$ corela.4754

ISSN : $1638-573 \mathrm{X}$

\section{Éditeur}

Cercle linguistique du Centre et de I'Ouest - CerLICO

\section{Référence électronique}

Viviane Arigne, «The boxer sleeps. Un générique particulier : le générique intitulatif », Corela [En ligne] 13-2 | 2015, mis en ligne le 09 janvier 2017, consulté le 20 avril 2019. URL : http:// journals.openedition.org/corela/4754; DOI : 10.4000/corela.4754

Ce document a été généré automatiquement le 20 avril 2019

\section{(c) (i) (2)(2)}

Corela - cognition, représentation, langage est mis à disposition selon les termes de la licence Creative Commons Attribution - Pas d'Utilisation Commerciale - Partage dans les Mêmes Conditions 4.0 International. 


\title{
The boxer sleeps. Un générique particulier : le générique intitulatif
}

\author{
Viviane Arigne
}

\section{Introduction}

1 Cette étude examine un aspect de l'expression de la généricité par le syntagme nominal défini singulier, dans un cas où ce syntagme fonctionne comme sujet dans une phrase extraite d'un texte de fiction anglophone The boxer sleeps. La phrase entière est rapprochée des titres de tableaux tels qu'ils sont étudiés ailleurs dans le cadre d'une étude pragmatico-discursive (Bosredon, 1997). La phrase étudiée n'est toutefois pas utilisée dans le contexte pragmatico-discursif canonique d'un titre de tableau, mais figure au sein du monologue intérieur d'un personnage, dans un discours rapporté libre, en dehors de toute représentation picturale. L'ensemble du travail est une analyse de discours, qui s'appuie sur une sémantique des référents construits (cf. par exemple et entre autres Kleiber, 1999, Arigne, 2005a) dans laquelle on distingue entre types et occurrences. Les analyses comportent une dimension contrastive, dans la double mesure où, d'une part, l'étude menée sur l'anglais trouve son étayage dans un travail mené sur des titres français et, d'autre part, l'interprétation et l'utilisation sémantico-discursive de certains formats morpho-syntaxiques se trouve être, en français et en anglais, identique ou assez voisine.

\section{Esquisse d'une problématique}

\section{1. La question de la référence}

Ce cas bien particulier de syntagme nominal (SN) défini singulier est donc un exemple attesté que l'on trouve dans une phrase extraite d'un texte de fiction. Cette phrase a déjà fait l'objet de commentaires qui concernaient, non pas le SN défini sujet, mais la forme verbale utilisée, qui est le présent simple. C'est pour cette raison que je vais aborder ce 
cas à partir du commentaire qu'il a suscité et donc par le versant verbal, verbe et nom étant, on le verra, indissociablement liés dans ce cas de figure. L'extrait en question, tiré d'une nouvelle de Tennessee Williams et présenté dans un ouvrage à visée didactique (Delmas et al., 1992), met en scène le personnage central Luke et un jeune Noir qui vient de livrer un combat de boxe, tous deux voyageant dans un bus à destination de Memphis. Il comporte la présentation de trois syntagmes nominaux comportant tous un nom discret $(\mathrm{Nd})^{1}$ : d'abord The young Negro (committed himself to sleep), puis The Negro ( represented) et enfin, au sein de la phrase qui est l'objet de la présente étude, The boxer ( sleeps).

(1) The young Negro committed himself to sleep as to battle, snoring upon a long rasping note, his shoulders swaying rhythmically against Luke's, his bandaged hand dangling between his legs. Luke regarded him with admiration and envy. The Negro represented, he thought, something splendid and heroic. Something that made life possible under any circumstances. A kind of impregnable simplicity. A completeness. An undividedness. The boxer sleeps, thought Luke. An exact statement. Said all that was necessary to say. (T. Williams, Ten-Minute Stop, in Delmas et al., $1992:$ 70, $1993: 41$ )

3 Cet exemple, proposé dans le chapitre 2 consacré au groupe verbal, est donné dans l'édition de 1992 comme sélectionné et commenté par Lancri, l'un des co-auteurs de l'ouvrage. Commentant cet emploi du présent simple, Lancri écrit :

" [...] la forme simple surprend. Pourquoi une forme simple surgit-elle, à la manière d'une information brute et première, alors que des éléments du contexte ont déjà mis en relation la source et le verbe en question? [...] avec l'emploi d'une forme simple, et qui plus est d'un présent, l'énonciateur donne à penser que cette réalité lui résiste et qu'elle ne se laisse pas analyser. [...] Malgré ses tentatives de commentaire, l'énonciateur ne dépasse pas le stade de la simple observation ». (Lancri, in Delmas et al., 1992 : 71, 1993 : 42)

4 Il s'agit là d'une analyse qui, fondée sur la partition adamczewskienne en phases 1 et 2 (Adamczewski, 1982: 37-77), cherche à assigner une interprétation invariante à chacun des termes de l'opposition syntaxique forme simple vs forme progressive. Il parait pourtant difficile ici de défendre l'idée selon laquelle, avec cet emploi de The boxer sleeps, l'on ne dépasserait pas «le stade de la simple observation ", qui semble être aussi celui d'une « relation première / primaire [...] coïncidant aussi parfaitement que possible avec la réalité extra-linguistique » (Lancri, in Delmas et al., 1992:72, 1993:42). On voit ici à l'œuvre une certaine conception de la problématique de la référence qui semble ne concevoir qu'un seul type de réalité extra-linguistique. Au contraire de la «simple observation » d'une entité tri-dimensionnelle s'offrant à la perception dans une situation spatio-temporelle, il semblerait plutôt que l'on ait affaire, avec The boxer sleeps, à une élaboration sophistiquée à partir de ce qui est, justement, observé avec The young Negro et qui se trouve dépassé par ce que cet objet d'observation représente (represented) et qui est un autre référent désigné par The boxer. Ce n'est donc pas tant que «la réalité [...] ne se laisse pas analyser ", mais bien plutôt que la réalité dont il s'agit avec The boxer est tout autre.

5 Il convient alors sans doute de distinguer deux plans et deux « réalités » différentes ayant chacune une ontologie différente et donc, en fin de compte, deux plans de référence distincts comportant chacun des référents distincts. Dans cet exemple, le syntagme The boxer ne donne pas la même référence que le syntagme The young Negro, et l'analyse du présent simple ne saurait valoir sans être, à mon sens, reliée à celle du syntagme nominal. 
Il s'agit bien ici de référence et d'un questionnement sur l'entité extra-linguistique à laquelle renvoie l'unité linguistique qu'est le $\mathrm{SN}$, et non simplement d'un sens référentiel qui ferait que le sens du nom Negro est différent de celui du nom boxer, ce sur quoi l'on peut imaginer que tout le monde s'entend ${ }^{2}$. Afin d'éviter toute équivoque, je rappellerai ici que les « réalités » mentionnées plus haut concernent des référents construits, dans une approche théorique (Kleiber, 1999, Arigne, 2005a) où la réalité est le fruit d'une conceptualisation par le langage. Il devient alors difficile de parler, comme le fait Lancri, de coïncidence, même plus ou moins parfaite, du langage avec la réalité.

\section{2. Le verbe et le nom : quelques manipulations}

Comme cela a été évoqué plus haut, Lancri pose des questions sur la forme verbale de présent simple et n'en pose pas sur le SN sujet. Je vais ici essayer de montrer que l'interprétation du nom et celle du verbe ont toutes deux à voir avec l'interprétation particulière de la phrase entière en proposant, dans une première étape, des manipulations faites indépendamment, sur le verbe d'une part, et sur le SN de l'autre.

7 Je commencerai par le verbe et les problèmes verbaux, abordés par l'auteur. On peut tenter de substituer deux formes à ce présent simple : d'une part une forme progressive, ce qui est l'objet de la discussion de Lancri ou, d'autre part, un prétérit puisque l'on est dans un discours rapporté au passé qui nous informe de ce que Luke se disait (thought Luke) à un moment qui s'insère dans un récit d'événements posés dans le passé. Le prétérit peut alors se présenter associé à la forme progressive ou ne pas se combiner à la forme progressive, donnant dans ce dernier cas ce que l'on appelle un prétérit simple. Ainsi, à partir de :

(1) [...] The Negro represented, he thought, something splendid and heroic. Something that made life possible under any circumstances. A kind of impregnable simplicity. A completeness. An undividedness. The boxer sleeps, thought Luke. An exact statement. Said all that was necessary to say.

on obtiendra :

(1a) ?The boxer is sleeping, he thought. An exact statement. Said all that was necessary to say.

(1b) ?The boxer slept, he thought. An exact statement. Said all that was necessary to say.

(1c) ?The boxer was sleeping, he thought. An exact statement. Said all that was necessary to say. ${ }^{3}$

Dans les trois cas (1a), (1b) et (1c), on se rend compte que les phrases proposées après manipulations ne sont pas dotées d'une bonne acceptabilité aux niveaux discursif et pragmatique. En effet, elles ne sont pas en adéquation avec le contexte, au sens où les commentaires qui suivent, An exact statement. Said all that was necessary to say, ne paraissent plus du tout adaptés et ne sont plus compatibles avec les nouvelles formes verbales proposées. Le détail des raisons de cette inadéquation apparaîtra dans la suite de la discussion et sera récapitulé en 3.1.

10 Comme je l'ai suggéré plus haut, on a le sentiment que l'interprétation du présent simple se trouve peut-être liée, dans cet exemple, à l'interprétation qui peut être faite du SN défini sujet The boxer. Il est également possible de tester ce SN et, suivant la direction amorcée par l'interprétation de Lancri qui voit sous ces deux SN le même référent et la même " réalité extra-linguistique », lui substituer The Negro. On obtient alors : 
(1d) The Negro sleeps, he thought. An exact statement. Said all that was necessary to say.

11 phrase dans laquelle le présent simple, parce qu'il est ressenti comme étant en harmonie avec le contexte à droite, convient toujours parfaitement, mais où le SN sujet peut susciter des discussions qui ne sont pas forcément proprement linguistiques et sont, justement, révélatrices de l'interprétation qu'il convient de donner à un tel SN sujet. Je reviendrai plus loin (2.4) sur cette question. Enfin, si les deux SN ont le même référent, on peut tenter de remplacer le premier SN présenté dans l'extrait The (young) Negro par un anaphorique :

(1e) The Negro represented, he thought, something splendid and heroic. Something that made life possible under any circumstances. A kind of impregnable simplicity. A

completeness. An undividedness. $\underline{\text { He }}$ sleeps, thought Luke. An exact statement. Said all that was necessary to say.

Il semble alors que l'absence d'autonomie référentielle du pronom he enlève à la phrase en question un élément qui, dans The boxer sleeps, parait jouer un rôle non négligeable dans l'interprétation de la phrase entière et qui est justement The boxer, le SN sujet.

\section{Le titre de tableau : une phrase-titre}

L'analyse du présent simple et celle du SN sujet semblant devoir être reliées, c'est donc la phrase entière et donc la proposition The boxer sleeps que je vais maintenant prendre en considération, la rapprochant d'une légende picturale ou d'un titre de tableau.

\section{1. Titres de tableaux et plans de référence hétérogènes}

14 Je m'appuierai pour cela sur les travaux de Bosredon (1997) qui, dans son ouvrage portant sur le français et intitulé Les titres de tableaux, analyse le titre de peinture comme se situant « entre légende et nom propre » (Bosredon, 1997 : 93). La formulation souligne au passage que le titre de peinture ne saurait être assimilé à une simple légende ${ }^{4}$. Toujours suivant les propositions de Bosredon, la phrase The boxer sleeps constituerait ce qu'il appelle une phrase-titre (Bosredon, $1997:$ 88), c'est-à-dire une phrase entière employée comme titre. Il s'agit par exemple de titres tels que Saint Bernard prêche la deuxième croisade (Emile Signol, 1840), La petite rêve (Paul Gaughin, 1881), ou encore, cité par Bosredon, Sylvie guérit Philis de la piqûre d'une abeille (François Boucher, 1755) ${ }^{5}$. Ces phrases-titres sont particulièrement intéressantes parce qu'elles utilisent un verbe de forme finie propre à construire une référence temporelle, à la différence d'autres titres qui, soit n'utilisent que des formes non finies pour l'essentiel participiales (cf. 2.3 infra), soit ne font pas figurer de verbe du tout ${ }^{6}$. De tels titres-phrases existent en anglais, tels que Ulysses mocks Polyphem (William Turner, 1829) ou The Warrior Sleeps (John Etherington, contemporain), cités plus loin.

15 Analysant la temporalité exprimée par le verbe, Bosredon est amené à s'intéresser, entre autres choses, à la relation entre temporalité et prototypie ainsi que, de manière plus générale, à la relation entre titres et prototypie. S'agissant de cette relation entre titres et prototypie, il écrit que les titres « construis[e]nt une représentation sémantique centrée, c'est-à-dire prototypique » (1997: 81) et, dépassant momentanément la problématique restreinte de la phrase-titre, il pose l'hypothèse pragmatique selon laquelle «l'intitulation guide la compréhension de la peinture, et réciproquement, la peinture sert 
à préciser le sens d'un titre » (Bosredon, 1997 : 81). L'existence d'un tel lien interprétatif, de nature pragmatique, entre le titre et la peinture, le conduit à questionner les problématiques conjointes de la référence et de l'accès aux référents dans le cas le plus difficile qui est celui de la peinture figurative. Ainsi, dans un cas de peinture figurative tel que

(2) Portrait de Jacqueline aux bras croisés / Jacqueline les bras croisés (Pablo Picasso)

16 «[...] le titre tantôt désigne à travers la figuration l'objet extra-pictural source de la représentation, tantôt la dépiction elle-même » (Bosredon, 1997: 77). L'on voit que la problématique de l'accès aux référents est rendue complexe dans la mesure où l'on a affaire, dans le cas de la peinture figurative, à deux types de référents bien distincts appartenant chacun à un plan de référence particulier. Une chose remarquable est que chacun de ces plans de référence, hétérogènes, traite la temporalité d'une manière différente. Le titre, quant à lui, donne accès simultanément aux deux plans de référence. Comme l'écrit Bosredon:

"L'accès au référent varie [...] selon que les prédicats caractérisants sont interprétables comme des prédicats coextensifs à l'objet-modèle ou coextensifs à la figuration ce qui conduit à des valeurs temporelles différentes : d'un côté on trouve des référents caractérisés par une propriété permanente, de l'autre des prédicats caractérisés par une propriété provisoire attestée par la figuration. Le titre est capable de désigner son référent dans les deux systèmes en même temps. Mais le lecteur peut oublier ce télescopage de deux espaces hétérogènes et croire à leur confusion. [...] Dans le référentiel de la toile, l'opposition permanente / provisoire disparaît. La saisie référentielle d'un morceau d'espace-temps ne se pose pas puisque la dimension temporelle n'appartient pas à ce système de repérage. Dans ces conditions, la propriété non permanente du point de vue de l'objet externe (mondain, extra-pictural...) se change en propriété permanente du point de vue de l'objet de représentation ». (Bosredon, 1997 : 78-79)

17 Ainsi en (2), la propriété accidentelle et non permanente est « avoir les bras croisés », de même que l'on aurait, de manière analogue, une propriété non permanente avec les exemples

(3) Nus assis / Nus couchés / Nu aux bras levés (Pablo Picasso)

18 cités par Bosredon (Bosredon, 1997 : 78) et dans lesquels cette propriété non permanente serait marquée par assis, couchés et aux bras levés. A côté de ce caractère non permanent et accidentel, le « système de repérage de la peinture » fait que « toute propriété exprimée par le titre s'interprète nécessairement comme une propriété permanente et définitoire de la figuration» (Bosredon, 1997 : 79). Ce caractère permanent donné par le titre se vérifie aisément à la faveur de l'examen de la peinture abstraite, avec laquelle toute dualité de référence se trouve abolie et "toute dépiction ne peut renvoyer qu'à ellemême» (Bosredon, 1997 : 79).

\section{2. The boxer sleeps : interprétation générique de la réalité picturale}

19 Dans tous les cas ayant à voir avec la peinture figurative, il convient donc de distinguer deux plans, qui sont d'un côté le plan de l'objet externe extra-pictural et, de l'autre côté, le plan de la représentation par l'image. Dans l'exemple étudié, les deux plans différents sont, d'une part, le plan du référent de The young Negro décrit par le narrateur et qui, 
venant de disputer un combat, s'assoupit et ronfle bruyamment et, d'autre part, le plan du boxeur endormi évoqué par la séquence The boxer sleeps qui, elle, fait partie du discours intérieur du personnage Luke. Si l'on a bien, dans les deux cas, un syntagme nominal de format $<$ the $+\mathrm{Nd}$ singulier $>$, la référence de ce syntagme $<$ the $\mathrm{Nd} \mathrm{sg}>\mathrm{est}$ différente dans chacun des deux cas dans la mesure où le syntagme The boxer a une interprétation générique que n'a pas le syntagme The young Negro dont le référent est bien ce jeune Noir endormi et en train de ronfler. L'interprétation générique de ce syntagme est liée au fait qu'il fait partie de la séquence The boxer sleeps qui doit être entendue ici comme un titre de tableau. Ce jeune homme en train de dormir est ici, pour reprendre les termes de Bosredon, doté d'une "propriété non permanente» qui est l'activité de sommeil, cette propriété se muant en propriété permanente pour l'«objet de représentation » qu'est The boxer dans The boxer sleeps. En résumé, le référent de The young Negro fait partie d'une réalité extra-linguistique offerte à la perception, et ce référent sert de support à la construction d'une autre réalité, tout aussi extra-linguistique, qui est une représentation et appartient à un autre plan de référence. Le commentaire cité in Delmas et al. (1992) évoque bien la "réalité extra-linguistique » mais il semble qu'il ne s'agisse pour l'auteur que celle qui s'offre à « la simple observation » et soit donc de l'ordre d'un réel tangible tridimensionnel s'offrant à la perception, aucun autre type de réalité n'étant explicitement envisagé.

20 S'agissant toujours de la peinture - et plus précisément de la peinture figurative, seule pertinente pour le cas étudié ici - et des problèmes temporels qu'elle peut poser, le titre nous donne, comme on l'a vu sous une formulation légèrement différente (2.1, supra) «une interprétation sémantique centrée, c'est-à-dire prototypique de ce que le titre exprime» (Bosredon, 1997 : 81)7. Ceci est vrai même si, ce qui est souvent le cas, écrit Bosredon, dans la peinture moderne post-classique, «[l]es propriétés visibles de la figuration picturale ne correspondent pas toujours à des propriétés de nature protypique » (Bosredon, 1997: 81). Il évoque ainsi le cas des lithographies de Henri Matisse regroupées sous le titre :

(4) Dix danseuses

21 qui, lorsqu'elles représentent, par exemple, des danseuses au repos, ne donnent pas à voir « un moment saillant et représentatif du référent ». On retrouve le même problème avec le tableau Danseuse Créole dans lequel, sans titre, il serait encore plus difficile de voir, au premier abord, une danseuse.

22 Si l'on accepte l'idée que cette phrase The boxer sleeps fonctionne, dans le discours dans lequel elle est insérée, à la manière d'un titre de tableau, la phrase entière The boxer sleeps aussi bien que son $\mathrm{SN}$ sujet The boxer nous donneront une interprétation prototypique, et pour le $\mathrm{SN}$, une interprétation générique qui renvoie au type que, dans une première approximation ${ }^{8}$, nous donnerons comme le type décrit ailleurs dans la littérature (Zemach, 1970 / 1990: 74, Galmiche et Kleiber, 1994:51, 1996:26, Arigne, 2005a: 26, $2005 \mathrm{~b}: 83,2006$ : III). Dans tous ces écrits, le type est posé comme une entité abstraite qui s'oppose à l'occurrence. Cette interprétation générique du syntagme nominal rejoint ce que Dubos (1990: 158) appelle la "valeur "emblématique" du prototype ». On remarque en outre ici que, par la formulation The Negro represented..., le texte de fiction rend manifeste une partie du processus d'élaboration propre à la représentation. De la même manière, il explicite le résultat de cette élaboration et construction mentale dans la séquence The boxer sleeps où The boxer renvoie au type et, plus précisément, à un type bien particulier qui est celui qui est associé à un objet de représentation picturale. Liée à la 
valeur prototypique du titre, cette valeur de type du syntagme nominal sujet se trouve, dans cet exemple particulier, indissociable de l'interprétation qu'il convient d'accorder au présent simple. La " propriété non permanente du point de vue de l'objet externe » se changeant en "propriété permanente du point de vue de l'objet de représentation " (cf. 2.1 supra), on a forcément un présent qui, pour rendre ce qui en français est un présent de l'indicatif, se trouve être en anglais un présent simple. En anglais, en effet, le présent simple fonctionne avec le présent progressif dans une opposition marqué vs non marqué, dont il est le terme non marqué. On comprend alors mieux que le présent simple, terme non marqué, soit le mieux à même de rendre le titre «capable de désigner son référent dans les deux systèmes en même temps " (Bosredon, 1997 : 78-79, cité plus haut en 2.1).

\section{3. Le verbe dans les titres anglais : le présent simple et le participe présent}

Ces dernières remarques sur le verbe conduisent à examiner d'autres formes verbales que l'on peut rencontrer dans des titres de tableaux en anglais. Les cas les plus typiques en anglais sont, comme cela est mentionné plus haut, le présent simple, et aussi la forme non finie de participe présent ${ }^{9}$. En effet, une phrase contenant un verbe au présent simple joue bien le rôle de titre de tableau comme en témoignent les titres suivants donnés sous forme de phrases :

(5) The Princess Shares her Dinner with the Frog (Walter Crane)

(6) Hannibal exceeds the Alps (William Turner)

(7) Ulysses mocks Polyphem (William Turner)

et il est par ailleurs possible d'imaginer un tableau ou une sculpture intitulé The Boxer Sleeps. En même temps, dans la mesure où cette phrase est un titre, on peut imaginer d'autres cas où le verbe ne serait pas au présent simple et ne serait pas non plus associé à une catégorie verbale finie. De fait, à côté de The Boxer Sleeps, l'on peut aisément concevoir, sans toutefois donner les deux formulations comme strictement équivalentes :

(8) The Sleeping Boxer

exactement comme l'on a, chez John Constable, The Leaping Horse (c. 1825). On note que le français pourrait très bien avoir ici, avec un participe passé, Le boxeur endormi ou, même, sans article et à la manière de Déjeuner sur l'herbe (Edouard Manet, 1863), Boxeur endormi ${ }^{10}$. Si l'on considère que The Leaping Horse a pour traduction conventionnelle Le saut, il est également permis d'orienter l'intitulation vers une autre formulation qui serait Le sommeil du boxeur. Un titre comme (8) ou encore

(1f) The boxer sleeps / He sleeps ${ }^{11}$

pourrait alors avoir comme équivalent français Le sommeil.

Il faut ici remarquer que les titres d'œuvres d'art telles que les tableaux et les sculptures ne sont pas les seules séquences à préférer le présent simple pour décrire une réalité visuelle. Il en va de même de nombre de commentaires d'actualité, sportifs ou autres, et des légendes photographiques. L'on peut ainsi citer l'exemple d'une phrase au présent simple, donné par Bouscaren et Chuquet (1987: 13) hors contexte et que l'on entendra comme un commentaire en direct d'un match de football :

(9) Rocheteau kicks the ball. 
Les auteurs proposent comme traduction française Coup de pied de Rocheteau, expression nominale de type $S N$ de $S N$, que l'on rapprochera aisément de la séquence Le sommeil du boxeur suggérée plus haut comme reformulation française de The boxer sleeps. Il y aurait donc une similitude formelle entre les titres de tableau et les commentaires sportifs, dans la mesure où l'on a, en anglais, le présent simple et, en français, la possibilité d'une équivalence donnée par le même format $S N$ de $S N$. Malgré ces similitudes formelles, il convient tout de même de se garder d'analyser ces deux types de phrases comme équivalents en termes d'interprétation. En effet, si la phrase Rocheteau kicks the ball devait être insérée dans le contexte, au passé, qui est celui de The boxer sleeps, on aurait :

(10) Rocheteau represented, he thought, something splendid and heroic. [...] Rocheteau kicks the ball, thought Luke. An exact statement. Said all that was necessary to say.

La phrase Rocheteau kicks the ball se verrait alors donnée comme an exact statement ou une formulation nécessaire (necessary to say), ce qui serait une interprétation difficilement tenable dans ce type de discours plus ordinaire qu'est le commentaire sportif, avec lequel on n'a pas affaire à la construction d'un type. On observe des choses analogues avec les légendes de photographies, lorsqu'il s'agit de simples photographies qui ne se veulent pas des photographies artistiques. Là encore, la légende ne saurait donner l'idée de nécessité qui est marquée par necessary (to say) ou celle de quelque chose d'essentiel suggérée par completeness et undividedness. On trouve ici, en restant dans le domaine sportif :

(11) PHOTOS (BLACK \& WHITE): Above: The referee's gloves ward off blood and the diseases it can carry. Below: This is the hard hour before a fight. The boxer rests, concentrates, tries to control his anxiety along with his breathing. When he sits up, his manager will smile and pretend absolute confidence. (COCA, Mother Jones, 1993)

Les choses sont bien entendu un peu plus ambiguës lorsque la photographie se donne des ambitions artistiques, ne serait-ce que dans les titres qu'elle adopte. La légende de la photographie se rapproche alors du titre de tableau, comme l'illustrent les intitulés cidessous :

(12) Pedro, The warrior rests <caliope.deviantart.com $>$ 12

(13) The warrior rests and ponders <gallery.shaviv.org>

On remarquera que ces deux légendes, qui accompagnent la photographie d'un enfant, tentent de dépasser la réalité saisie par l'objectif pour en proposer une lecture symbolique dans laquelle cet enfant est vu comme un guerrier ( $a$ warrior). Dans cette tentative d'accéder à la dimension symbolique de l'art, ces deux légendes photographiques rejoignent le titre d'un tableau représentant le cuirassé HMS Warrior, aujourd'hui devenu bateau-musée et maintenu à quai à Portsmouth de façon permanente :

(14) The Warrior Sleeps (John Etherington, dit John e) <paintingsilove.com>

On observe avec ce titre de tableau contemporain la même utilisation du présent simple sleeps que dans la phrase de fiction qui constitue l'objet d'étude de cet article The boxer sleeps. 


\section{4. Le syntagme nominal sujet dans la phrase-titre : la question du nom}

33 A la suite de ces considérations sur le verbe des titres de l'anglais, je proposerai maintenant quelques remarques sur l'interprétation du SN sujet présent dans les phrasestitres. Ces remarques valent pour tous les SN définis comportant un $\mathrm{Nd}$ singulier, en anglais comme en français. L'on se souvient que, dans l'hypothèse où les deux syntagmes seraient vus comme référentiellement équivalents et où l'interprétation des deux SN The Negro et The boxer se réduirait à la seule fonction consistant à désigner l'objet extrapictural (cf. 2.1 et 2.2) qui est ce jeune homme qui vient de disputer un combat et serait, dans le cas d'une peinture, le modèle, on pourrait sans aucune difficulté avoir la substitution proposée précédemment (1.2) en (1d) :

(1d) The Negro sleeps, he thought. An exact statement. Said all that was necessary to say.

Cela n'est pas le cas dans le contexte considéré, dans lequel cette substitution apparaît curieuse et, pour certains, insultante, dans la mesure où le SN The Negro construirait alors un type, associé à un tableau qui se voudrait une représentation artistique et symbolique de ce qu'est un Noir endormi. On aurait alors un titre de tableau équivalant en français à Le sommeil du Noir / du Nègre ou encore de Nègre endormi, ce qui pourrait vouloir dire que le type nominal qui, dans ce contexte, fonctionne comme référent, serait construit à partir de traits essentiels associés au nom Negro et non au nom boxer, ou que quelque relation tout aussi essentielle associerait le Nègre et son sommeil pour donner au titre entier une valeur prototypique. C'est d'ailleurs ce que suggère, sous une forme légèrement différente avec forme verbale non finie, le titre de tableau :

(15) The Sleeping Negro (Langston Hughes, Slave on the Block, 1933)

Il s'agit du titre qu'un personnage de fiction d'une nouvelle de Langston Hughes, Anne Carraway, souhaite donner à la toile qu'elle s'apprête à peindre. Dans ce cas précis, la relation essentielle entre le Nègre et son sommeil se trouve explicitée dans le monologue intérieur du personnage qui suit immédiatement la mention du titre de tableau: Dear, natural childlike people, they would sleep anywhere they wanted $t^{13}$. Il est par ailleurs sans doute significatif que, dans cette construction d'un type associé à un titre de tableau, ait été choisi The boxer, séquence comportant un nom d'animé humain qui renvoie à l'argument sujet d'un verbe dont ce nom est, justement, dérivé. On se rapproche là d'une catégorie de tableaux comportant des intitulés exclusivement nominaux, singuliers ou pluriels, comme :

(16) Les baigneuses (Gustave Courbet), Danseuses (Edgar Degas), Les glaneuses (Jean-François Millet), La liseuse (Claude Monet), Le semeur, Les buveurs (Vincent Van Gogh)

Dans de tels titres, « le centrage de la représentation apparait particulièrement évident » (Bosredon, 1997 : 81) et se voit également lié à la question de la temporalité, puisque le semeur est montré en train de semer, la liseuse en train de lire et les baigneuses en train de se baigner, alors que le boxeur, dans l'exemple considéré ici, n'est pas vu en train de boxer. C'est d'ailleurs fort probablement pour cela que certains des informateurs anglophones interrogés ont ressenti le besoin d'expliciter leur interprétation par la locution adverbiale at last (The boxer sleeps at last) qui permet, par raccroc, de récupérer dans l'interprétation l'idée de l'activité même du boxeur ${ }^{14}$. 


\section{Le titre-phrase}

Le fait que les séquences The Negro sleeps, Rocheteau kicks the ball ou encore He sleeps soient difficilement concevables dans le contexte en question, va peut-être apparaître plus clairement dans cette troisième partie consacrée, non plus à la phrase-titre, mais au titrephrase.

\section{1. Titre et nom : le titre-phrase}

La possibilité de titres alternatifs qui sont des syntagmes nominaux comme The sleeping boxer ou, en français, Le sommeil du boxeur (2.3), attire l'attention sur les similarités que peuvent avoir les titres de tableau au présent, c'est-à-dire des titres qui sont des phrases entières, et le nom. Bosredon y consacre un long paragraphe qu'il intitule « Titre-phrase au présent » (Bosredon, 1997 : 88-90). Dans sa discussion, il commente ainsi la parenté de cet emploi particulier du présent de l'indicatif français et du nom :

«Le présent, contrairement aux autres temps verbaux, est compatible avec toutes les époques ancrées dans et par l'énonciation. Il partage même avec les noms certaines propriétés référentielles; en l'occurrence la neutralisation des relations et rôles déictiques. Seul est activé le repérage de la désignation exprimé par le titre, repérage permettant à celle-ci de référer à un état de fait représenté, c'est-à-dire à la fois présent dans le monde du lecteur mais aussi absent de celui-ci. Temporellement débrayées, ces phrases n'ont plus qu'un rôle descriptif analogue à celui d'une suite désignative nominale ». (Bosredon, 1997 : 89)

Ces phrases au présent que sont certains titres peuvent donc être considérées comme des «strutures phrastiques à rôle dénominal » (Bosredon, $1997: 89$ ) et être rapprochées des didascalies qui caractérisent un "état de fait théâtral » ou, comme on l'a vu avec l'exemple (9) Rocheteau kicks the ball des commentaires sportifs ou encore, en (12), des légendes de vignettes encyclopédiques ou de photographies. De ce point de vue-là, «Le présent de l'indicatif du français doit donc être considéré d'une part comme une forme non temporelle en raison de sa capacité à référer dans toutes les époques, [...] En tant que capteur possible de tous les instants ou domaines d'instants par rapport à la prise de parole, il neutralise les relations construites dans le système de repérage déictique : il est donc un non-temps utilisable pour tous les repérages temporels ». (Bosredon, 1997 : 90)

Si l'on admet cette analyse, il devient possible d'affiner les arguments permettant d'expliquer que la forme qui, en anglais, correspond à cet emploi particulier du présent de l'indicatif français ne puisse être le présent progressif et soit le présent simple. Dans l'exemple analysé, l'utilisation de la forme progressive et de son interprétation imperfective :

(1a) The boxer is sleeping [...]

nous ferait quitter le plan de la représentation et nous placerait sur le plan de cette réalité qui, lourde de deixis, sert de support à la représentation. Cela ne permettrait pas de rendre la référence que donne le présent de l'indicatif français qui «[...] indique [...] l'existence d'un processus dans un cours des choses inassignable au plan déictique " (Bosredon, 1997:90). On notera que, d'un point de vue plus général, cette voie 
d'interprétation du présent n'est pas nouvelle puisqu'elle avait été abordée par Serbat (1988), dont Bosredon déclare s'être assez librement inspiré15. Se rapprochant du nom, le titre-phrase et donc aussi la phrase-titre sont une façon de symboliser et de styliser, d'épurer un état de choses initial afin de le placer sur un autre plan, plus abstrait, qui est celui de la représentation et de la construction esthétique. Dans l'exemple considéré, cette phrase qui constitue le titre est constituée d'un verbe au présent simple typique du générique ${ }^{16}$, auquel est associé un argument sujet sous la forme d'un $\mathrm{SN}$, renvoyant à un référent abstrait qui peut être analysé comme un type et a donc également une interprétation générique. Ce n'est bien sûr pas le cas de tous les titres, l'argument sujet pouvant avoir comme tête de syntagme un nom propre, que celui-ci soit utilisé seul comme Hannibal en (6) ou à l'intérieur d'un SN comme avec The Warrior en (14) ${ }^{17}$.

L'on se souvient (1.2) que les manipulations consistant à utiliser le progressif ou le prétérit ne parvenaient pas à restituer l'interprétation associée à The boxer sleeps dans l'exemple (1) considéré. En effet, elles échouent toutes à rendre l'interprétation de généricité de ce titre-phrase. La variation is sleeping de (1a) ne convient pas en raison de la présence d'une forme progressive d'interprétation trop déictique, tandis que la proposition (1b), faisant figurer le prétérit slept, donne ce dernier comme contraint par la concordance des temps du discours indirect libre : il rattache ainsi l'événement décrit au processus mental du personnage Luke et donc à une situation déictique très déterminée d'un point de vue spatio-temporel. On peut le deviner, la manipulation (1c) comportant was sleeping et cumulant prétérit et forme progressive, ne réussit pas davantage à rendre l'interprétation de l'exemple de départ. La quatrième modification The Negro sleeps proposée en (1d) conserve, potentiellement, la valeur de titre de tableau mais pose un problème pragmatique et sociologique dans la mesure où, on l'a noté (2.4), il s'agirait de Le sommeil du Noir / du Nègre ou encore de Nègre endormi ce qui n'est bien entendu pas ce qu'il faut entendre avec The boxer sleeps dans l'exemple considéré. Enfin, la manipulation (1e) He sleeps peine à constituer un titre dans un contexte uniquement langagier comme l'est ici celui de la fiction littéraire : l'absence d'autonomie référentielle de l'anaphorique fait que le seul pronom serait difficilement interprétable dans un titre sans la coprésence de l'objet qu'est l'œuvre d'art réelle, tableau ou sculpture ${ }^{18}$. On notera, pour conclure ce paragraphe, le caractère court de la phrase The boxer sleeps, son début par une majuscule sans qu'aucune coordination ne vienne l'imbriquer dans un enchaînement temporel narratif, ainsi que son caractère assertif ( $a$ statement), qui en font un titre-phrase à bien des égards exemplaire. Le caractère court et la majuscule sont respectivement préparé et imposée par les deux phrases averbales A completeness et An undividedness qui, brèves elles aussi, sont des phrases nominales ponctuées par un point final. Les deux SN renvoyant chacun à une unité discrète annoncée deux fois par la forme nominale thing fonctionnant comme formatif de something dans le contexte gauche, ces phrases nominales préparent également pour la phrase The boxer sleeps son interprétation de titre et son « rôle dénominal».

\section{2. Le titre, une image juste}

43 Je terminerai cette partie par quelques considérations générales sur le titre, qu'il s'agisse d'un titre-phrase ou non. Ces remarques peuvent sembler quelque peu secondaires par rapport à ce qui précède mais elles me semblent justifiées, dans ce cas précis, par le caractère explicite du contexte de la phrase The boxer sleeps. Mon point de départ sera ici 
une remarque que fait Bosredon sur le titre de tableau, en tant qu'il oriente vers une interprétation prototypique. L'auteur écrit :

«[...] quelle que soit l'esthétique dont elle relève, l'image en relation avec le titre est toujours exprimée par le titre comme une image particulièrement juste - même si cette justesse ne peut être elle-même justifiée que dans une analyse de nature esthétique ». (Bosredon, 1997 : 81)

L'image étant donnée comme en parfaite adéquation avec le titre, il est possible de poser que, de manière converse, le titre lui-même est en parfaite adéquation avec l'image proposée. On rejoindrait alors le texte de Tennessee Williams dans lequel le personnage de Luke donne The boxer sleeps comme an exact statement. Notons au passage que l'anglais exact, tout comme le français exact, a son origine dans le latin exactus, qui lui-même vient du verbe exigere au sens de achever : exact signifie alors " poussé jusqu'au bout, accompli » (Onions, 1966: 333, Robert, $1990: 719$ ). On a alors une idée de complétude ontologique (Arigne 2010, 2015 ${ }^{19}$ ) donnée par la notion de l'exactitude de l'expression, cette idée d'exactitude étant associée au titre et au statement qu'est le titre-phrase The boxer sleeps, et liée à une notion de nécessité exprimée dans ce qui suit par all that was necessary to say. $\mathrm{Si}$, enfin, l'on accepte de se laisser quelque peu dériver vers une explication de texte non linguistique, il peut être tentant de suggérer que c'est ce sentiment qu'a le personnage d'être en face d'une réalité essentielle ([a] completeness [...], [a]n undividedness [...]) qui le conduit à élaborer cette phrase-titre The boxer sleeps qui doit "encapsuler » au sens de l'anglais encapsulate et donc, finalement, incarner dans la langue la complétude ontologique qu'il lit dans le spectacle qui s'offre à ses yeux.

\section{Le générique artistique ou intitulatif : type ordinaire et type artistique}

Ces analyses de la phrase-titre et du titre-phrase ont permis d'avancer dans la compréhension de l'emploi du présent simple dans cette phrase de Tennessee Williams, en assimilant cette dernière à un titre donné par le personnage Luke à la représentation intérieure qu'il construit à partir de ce qu'il peut observer dans un monde tridimensionnel. Le SN défini singulier The boxer a été pour sa part relié à la généricité exprimée par la phrase du titre-phrase, et rapproché de l'expression d'un type (2.2 et 2.4). Il convient ici de revenir sur le SN défini singulier The boxer afin d'en affiner l'interprétation.

Ce sont donc, en résumé, la fonction de désignation et le rôle dénominal du titre-phrase, ainsi que son caractère a-temporel lié au plan représentationnel qui est le sien, qui permettent d'identifier dans ce syntagme nominal The boxer une valeur générique qui renvoie à un type. Dans cette idée de type, se rejoignent diverses analyses convergentes (2.2), dont celle de Galmiche et Kleiber (1994 / 1996) et celle de Bosredon (1997), qui évoque l'interprétation prototypique de valeur «centrée " donnée par le titre dans la peinture figurative. Ceci posé, la question est de savoir comment on peut définir ce type nominal associé au SN The boxer. En effet, il ne s'agit pas ici véritablement d'un type comme celui auquel renverrait, par exemple, le SN the Siberian tiger employé en zoologie, mais d'un type bien particulier que l'on pourrait qualifier d'artistique ou, dans une formule plus longue, de générique intitulatif de l'œuvre d'art visuelle. 

de la deixis et des occurrences. Contrairement au type linguistique ordinaire qui est un référent abstrait obtenu "par abstraction des particularités des occurrences » (Galmiche et Kleiber, $1994: 51$ / 1996: 26), le type artistique ou intitulatif présente la particularité d'illustrer ce référent abstrait par une représentation figurative unique et occurrentielle qui est celle de l'œuvre d'art, par exemple un tableau. Ce sont justement ces détails et particularités occurrentielles qui constituent non pas seulement le substrat mais aussi la matière de la construction du type et de la généralité exprimés par le titre et, dans ce titre, par le SN du titre. En d'autres termes, le type intitulatif de cette interprétation générique du $\mathrm{SN}$ ne peut exister sans la coprésence de l'occurrence, unique, qu'est l'œuvre d'art ${ }^{20}$, et la représentation ou « dépiction » (cf. 2.1 et Bosredon, $1997: 77-79$ ) que cette œuvre d'art propose. Il diffère en cela du type « ordinaire » que l'on a avec The dog ou the Boxer dans des exemples tels que :

(17) The dog is a mammal

(18) Weinberg, S. K. et Arond H., "The Occupational Culture of the Boxer", American Journal of Sociology, $\mathrm{n}^{\circ} 62$ (p. 460), mars $1952<$ <aboxe.net $>^{21}$

equel on a affaire à « des emplois abstraits, détachés des circonstances spatiotemporelles » (Galmiche et Kleiber, Ibid.) que l'on n'a pas à rapporter à une représentation occurrentielle particulière. En dépit de leur construction et visée différentes, ces deux types trouvent dans la langue, avec les noms discrets, la même expression formelle the $N d$ $s g$ faisant usage de la forme de singulier. Ce type abstrait «ordinaire» est doté d'une valeur générique, comme cela apparaît clairement dans l'exemple ci-dessous, au sens où cette interprétation générique est parfois dite renvoyer à l'espèce ou kind (cf. par exemple et entre autres Beyssade, 2005, Pelletier, 2010b) et, d'une certaine manière, à l'ensemble des occurrences pouvant recevoir l'appellation donnée par le nom discret. Dans l'exemple (19) ci-dessous, le renvoi à l'espèce ou kind se trouve explicité par le SN attribut the noble breed:

(19) The boxer is the noble breed, that I call them. They're simple kind of guys and they 're good-hearted kind of guys. I'm talking generally... (COCA, CNN, 2001)

En outre, la valeur générique y est explicitée par les reprises anaphoriques du SN défini singulier the boxer par le pronom pluriel de $3^{\mathrm{e}}$ personne they et commentée plus loin par l'adverbe generally. L'on notera enfin que c'est un générique "ordinaire» exprimant clairement la multiplicité par le pluriel (they) et un nom collectif (people), qui sert de substrat à la création du générique intitulatif, comme le montrait le contexte explicitant la généricité nominale et phrastique du titre de tableau The Sleeping Negro donné comme exemple (15) (cf. 2.4 et note 12).

Afin de clore cette discussion, je proposerai une dernière remarque concernant le contexte et l'environnement discursif de la phrase étudiée. Les considérations qui suivent ne sauraient avoir valeur de preuve mais, une fois encore, le contexte qui suit vient commenter et, d'une certaine façon, appuyer l'analyse du SN défini singulier The boxer comme entrant dans une séquence pouvant être un titre de tableau. En effet, l'effort de stylisation que j'ai dit être celui du générique intitulatif et l'idée de représentation artistique visuelle sont tous deux poursuivis dans la suite du contexte dans une seconde élaboration, plus abstraite, qui nous fait quitter le domaine de la peinture figurative. C'est ce que montre la phrase Luke [...] seemed to see his life in graphic design, qui suit l'extrait présenté en (1) :

Corela, $13-2$ | 2015 
(1bis) The young Negro committed himself to sleep as to battle, snoring upon a long rasping note [...] Luke regarded him with admiration and envy. The Negro represented, he thought, something splendid and heroic. [...] A kind of impregnable simplicity. A completeness. An undividedness. The boxer sleeps, thought Luke. An exact statement. Said all that was necessary to say. Luke glanced at the Negro and seemed to see his life in graphic design. A strong black line pushing stubbornly forward without a curve. (T. Williams, Ten-Minute Stop, p. 55, <books.google.fr>)

Les deux formulations glanced at et seemed to see permettent de distinguer à nouveau clairement, d'une part, un plan de référence que je qualifierais de «ordinairement déictique » et dans lequel on retrouve en objet direct le SN the Negro (cf. 1.1 et 2.2) et, d'autre part avec seemed to see, un deuxième plan de référence qui est celui de la représentation intérieure construite par le personnage Luke à partir de ce qui s'offre à sa $v_{u}^{22}$. Le dessin graphique (graphic design) imaginé qui représente la vie du jeune Noir est ensuite décrit comme une ligne épaisse et noire (a strong black line [...]). On note que cette partie du contexte n'était pas donnée dans l'exemple cité dans l'ouvrage de Delmas et al. (1992: 70, 1993: 41), et que la ligne épaisse et noire n'est pas, dans ce monologue intérieur, une entité extra-linguistique du monde tridimensionnel accessible aux sens du personnage Luke.

\section{Conclusion}

Au terme de cet examen, il est sans doute possible de dresser une petite récapitulation des acquis de la réflexion, présentée ci-dessous sous formes de points.

- Le type est un référent abstrait construit par le SN défini singulier de manière pragmatique (voir Declerck, 1991, pour les interprétations génériques plus ordinaires) et obtenu par une catégorisation fondée sur une stylisation, forme d'abstraction figurative. Cela est vrai pour les interprétations génériques des SN anglais et français the dog, the Siberian tiger, le chien, le tigre de Sibérie, ainsi que pour les titres de tableaux en anglais et en français La Liseuse, The Reader, the Sower. La possibilité d'une telle interprétation du SN défini singulier est liée au caractère discret des $\mathrm{N}$ utilisés. En effet, les $\mathrm{N}$ discrets désignent à la fois les occurrences et le type ou, dans certaines analyses, l'espèce ou kind.

- Il convient de distinguer le type ordinaire du type intitulatif caractéristique des œuvres d'art visuelles, ce dernier étant soumis à des conditions pragmatiques d'interprétation bien particulières (Bosredon, 1997).

- Le générique intitulatif a besoin de l'occurrence qu'est l'œuvre d'art pour être interprété. L'œuvre d'art n'est pas un objet dont le terme dénominatif fait d'emblée partie des connaissances qu'ont les locuteurs sur la langue, et elle est, dans les cas typiques, nommée par son seul créateur.

- La distinction, sous la même forme d'un SN défini singulier, de plusieurs types de types et, ainsi, plusieurs types d'interprétation générique, rappelle combien il est important de distinguer des plans de référence distincts. Il convient alors d'examiner les spécificités référentielles de ces référents d'ordre différent.

Une des leçons de cette étude est que, en matière de généricité comme dans bien d'autres, il faut sans doute se garder de... généraliser. « Généricité " pourrait d'ailleurs bien être un terme dont on peut se dispenser (Wilmet, 1988) et l'on pourrait aujourd'hui dire que, de la même manière qu'il existait une "nébuleuse de la modalité » (Meunier, 1981), il existe 
une nébuleuse de la généricité. Si l'on utilise le terme, il vaut donc mieux être conscient que son utilisation ne saurait passer pour une analyse et que le simple étiquetage d'un emploi comme générique laisse assurément plus de grain à moudre que de grain moulu. Il convient également de souligner combien il est crucial de prendre en compte dans les analyses linguistiques les spécificités référentielles. Il est assez vraisemblable que la langue, en tant qu'activité symbolique reposant sur des mécanismes cognitifs, travaille avec des outils assez élémentaires et peut-être même rudimentaires. Mais les choses dont elle parle et les référents qu'elle construit sont parfois extrêmement abstraits et il appartient au linguiste de décrire ces référents abstraits et cette abstraction. Certains linguistes s'y sont déjà intéressés, comme Bosredon (1997), dont l'ouvrage est abondamment cité ici, ou dans des domaines linguistiques plus traditionnels et pour ne citer qu'un seul auteur, Anokhina (2002), qui s'est attachée à décrire le mécanisme de référence des noms abstraits. Au-delà de la problématique de la généricité, cet article constitue une contribution à l'étude pragmatico-discursive des titres en général, titres de tableaux ou titres d'œuvres textuelles, de paragraphes ou de sections ${ }^{23}$. Enfin, ce travail montre ce que les études sémantiques et pragmatiques peuvent apporter à l'étude d'un discours de fiction littéraire. L'on voit comment un auteur de fiction, en l'absence de mention explicite d'un quelconque tableau ou titre de tableau, présente un titre suffisamment canonique pour pouvoir être interprété comme tel isolément ${ }^{24}$, tout en fournissant dans l'environnement discursif proche les éléments permettant de confirmer l'interprétation correcte de la formule de son personnage comme un titre de tableau ${ }^{25}$. En même temps, le discours littéraire tire parti de l'ambiguïté potentielle de référence du SN défini singulier qui, en raison de sa possible interprétation occurrentielle avec laquelle il se trouverait être co-référent du SN précédent The (...) Negro, permet d'articuler la phrasetitre et le titre-phrase avec la narration ${ }^{26}$.

ADAMCZEWSKI, H. 1982. Grammaire linguistique de l'anglais. Paris : Armand Colin (avec la collaboration de Cl. Delmas).

ANoKHINA, 0. 2002. Sur le mécanisme de référence des noms abstraits. Cahiers de lexicologie. Revue internationale de lexicologie et lexicographie $81: 39-49$.

ARIGNE, V. 2005a. Le syntagme N1 of N2 et la référence multiple des massifs anglais. Revue de Sémantique et Pragmatique 17 : 7-39.

ARIGNE, V. 2005b. Les discrets collectifs face aux massifs : des modes de discrétisation du massif », G. Girard (éd.) Parcours linguistiques. Domaine anglais, CIEREC, Travaux 122, Publications de l'Université de Saint-Etienne : 73-87.

ARIGNE, V. 2006. Les discrets collectifs face aux massifs : des modes de discrétisation du massif (version longue), J.-Cl. Souesme (dir.), Cycnos 23, $n^{\circ} 1$, Le qualitatif. http://revel.unice.fr/

ARIGNE, V. 2010. Subjectivité et référence. Questions de sémantique, dossier soumis pour l'Habilitation à diriger des recherches, Université Paris-Sorbonne (Paris 4), vol. 1 : Synthèse de l'activité scientifique http://hal-univ-paris13.archives-ouvertes.fr/hal-00691193

ARIGNE, V. 2015. La modalité appréciative : vers une sémantique de good. Anglophonia 19.

$<$ anglophonia.revues.org>

BEYSSADE, C. 2005. Les définis génériques en français : noms d'espèce ou sommes maximales. Noms nus et généricité, Presses Universitaires de Vincennes : 33-63.

BosRedon, B. 1997. Les titres de tableaux. Une pragmatique de l'identification. Paris : Presses Universitaires de France. 
BOUSCAREN, J., CHUQUET, J. 1987. Grammaire et textes anglais; guide pour l'analyse linguistique, Paris, Gap : Ophrys (avec la collaboration de L. Danon-Boileau).

ComRIE, B. 1976. Aspect. Cambridge : Cambridge University Press.

DECLERCK, R. 1991. The origins of genericity. Linguistics 29 : 79-102.

Delmas, Cl., AdAms, P., Deléchelle, G., Girard, G., LANCRi, A., NAudé, G. 1992. Faits de langue, faits de discours en anglais. La Garenne-Colombes : Editions de l'Espace Européen [1993. Faits de langue en anglais. Paris : Dunod].

DuBos, U. 1990. L'explication grammaticale du thème anglais. Paris : Nathan.

Flaux, N., Glatigny, M., SAmain, D. (éds). 1996. Les noms abstraits. Histoire et théories, Actes du colloque de Dunkerque, 15-18 septembre 1992, Villeneuve d'Ascq : Presses Universitaires du Septentrion.

Galmiche, M., Kleiber, G. 1994. Sur les noms abstraits. In G. Kleiber, 1994, Nominales, Essais de sémantique référentielle, Paris, Colin. 48-64 ; repris in N. Flaux, M. Glatigny, D. Samain (éds.), 1996, Les noms abstraits, Histoire et théories, Actes du colloque de Dunkerque, 15-18 septembre 1992, Villeneuve d'Ascq : Presses Universitaires du Septentrion. 23-40.

KLEIBER, G. 1994. Nominales, Essais de sémantique référentielle. Paris : Colin.

KLEIBER, G. 1999. Problèmes de sémantique. La polysémie en questions, Villeneuve d'Ascq : Presses Universitaires du Septentrion.

MEUnIER, A. 1981. Grammaires du français et modalités. Matériau pour l'histoire d'une nébuleuse. DRLAV 25, Dans le champ pragmatico-énonciatif : 119-144.

OnIONS, Ch. T. 1966. The Oxford Dictionary of English Etymology. Oxford : Oxford University Press.

Pelletier, F. (ed.). 1979. Mass Terms: Some Philosophical Problems, Dordrecht : Reidel.

Pelletier, F. (ed.). 2010a. Kinds, Things and Stuff. Mass Terms and Generics. Oxford, New

York, Oxford University Press.

Pelletier, F. 2010b. Generics: A Philosophical Introduction. In F. Pelletier (ed.)

Kinds, Things and Stuff. Mass Terms and Generics : 3-15.

Rebeyrolle, J., Jacques, M. P., Péry-Woodley, M. P. 2009. Titres et intertitres dans l'organisation du discours. Journal of French Language Studies, 19 (02) : 269-290.

ROBERT, P. 1990. Le Petit Robert 1. Dictionnaire alphabétique et analogique de la langue française.

SERBAT, G. 1988. Le temps : le temps des philosophes, des savants, des grammairiens..., et celui des sujets parlants. Linguistique latine et linguistique générale. Louvain-la-Neuve, Peeters, pp. 15-21.

ZEMACH, E. 1970. Four Ontologies. Journal of Philosophy, 62 : 213-247 ; repris in F. Pelletier (ed.), 1979, Mass Terms: Some Philosophical Problems, Dordrecht : Reidel. 63-80.

WiLmET, M. 1988. Contre la généricité. Lingua 75, 2-3 : 231-250. 


\section{NOTES}

1. Le nom discret s'oppose au nom massif en ce qu'il impose des limites à l'entité à laquelle il renvoie (Arigne, 2005a) et permet ainsi d'isoler une unité (Arigne, 2005b, 2006, contra 2005a). Le nom massif, quant à lui, est caractérisé par l'absence de limites données à l'entité à laquelle il renvoie.

2. Pour « sens référentiel », voir par exemple Kleiber (1999 : 15-52) ou Arigne (2005a : 12-14).

3. On notera qu'un modèle de prétérit simple analogue à celui de (1b) est fourni par les deux exemples de discours indirect libre suivants : They did talk nonsense, he thought, the Ramsays (V. Woolf, To the Lighthouse, 1958 / 1978, p. 105); She slept, he thought, or perhaps not, for when she began to cry and he felt her hot tears on his neck and when she began to sob and cling to him tighter and tighter, he could only hold her and hope her fear would pass (Amicus, Tiffany, 2010, books.google.fr).

4. La question sera abordée plus loin (2.3).

5. Bosredon (1997 : 88) cite également La petite s'amuse (Paul Gaughin, 1881), mais il convient de noter que cette phrase semble n'être qu'une partie du titre plus long généralement donné : La mare aux canards. La petite s'amuse.

6. Bosredon (1997 : 75-90) ne parle de phrase que dans le cas où le verbe est à une forme finie dans les « titres-phrases au présent » qui ne représentent que l'un des cas où le titre adopte « une forme d'énoncé ». Pour des raisons de commodité, cette acception sera suivie dans la totalité des analyses de titres de la présente étude (cf. 3.1 pour une prise en compte de phrases averbales qui ne sont pas des titres).

7. Une citation plus complète est «[...] la lecture du titre est communément orientée vers une interprétation sémantique centrée, c'est-à-dire prototypique, de ce que le titre exprime ». La citation donnée en 2.1 se trouve plus bas sur la même page (Bosredon, 1997 : 81).

8. Cette notion de type concernant ce cas particulier de titre de tableau sera discutée plus loin en 4.

9. Les formes françaises correspondantes sont le présent de l'indicatif et, aussi, le participe présent. Pour ce dernier cas, voir Bosredon (1997 : 83-87).

10. On notera que La Bohémienne endormie, titre d'un tableau de Henri Rousseau (1897), a pour traduction conventionnelle The Sleeping Gipsy, La fileuse endormie de Gustave Courbet (1853) est rendu par The Sleeping Spinner et le titre sans article du tableau Enfant endormi de Paul Gaughin (1884) devient en anglais The Sleeping Child. L'on remarque également que le titre proposé en (7) Ulysses mocks Polyphem (William Turner (1829) est souvent donné sous la forme Ulysses deriding Polyphemus.

11. Je remercie Eithne O'Neill, qui m'a signalé un tableau de Thomas Eakins représentant un boxeur et auquel l'artiste a attribué le titre latin Salutat (1898). Les commentateurs et critiques proposent tous comme traductions anglaises possibles He greets ou He salutes comportant le présent simple. Ceci est l'occasion de noter que, même si les verbes salute et sleep présentent des propriétés différentes en termes d'Aktionsart, ils n'interdisent pas l'utilisation de la forme progressive qui permettrait, pour le procès auquel ils renvoient, une description aspectuelle imperfective (Comrie, 1976) qui serait celle d'un point ou portion quelconque du déroulement. Cette forme n'a pas été choisie.

12. Tous les liens cités dans cet article ont été consultés sur la période de juin-juillet 2015.

13. Une traduction française publiée est Chère race enfantine et naturelle, ils dormaient n'importe où (Hélène Bokanowski, 1990). L'on voit ici comment le texte de fiction, par la voie d'un monologue intérieur, propose un commentaire de ce titre de tableau, en reliant l'idée d'un sujet générique ( 
people, they) et celle d'un comportement (sleep) attendu pour le référent du sujet en toute occasion (would, anywhere) et donc générique également sur le plan spatio-temporel. On notera aussi le titre français Nègre endormi d'une sculpture de Philippe Solari (1868), souvent rendu en anglais par Sleeping Negro.

14. L'on notera au passage que les traductions anglaises du titre du tableau Le sommeil, de Gustave Courbet (1866), hésitent entre Sleep et The Sleepers (pour un titre tel que Le sommeil, voir aussi 2.3 supra). S'agissant du lien entre l'activité du référent du nom argument sujet (cf. 2.4) et le terme de cette activité, ici suggéré par le verbe sleep, on lira avec intérêt les considérations de Bosredon (1997 : 77) sur les cas possibles, dans les titres, de saillance d'un «dernier moment "valant comme l'expression d'un état résultant, ainsi que sur la multiplicité des valeurs aspectuelles que peut évoquer un titre associé à une représentation picturale figée qui, elle, ne peut donner à voir qu'un seul moment d'un procès. On aurait ici un cheminement inverse : c'est la propriété non télique du verbe sleep qui en ferait un état résultant renvoyant à l'activité antérieure signalée par le nom boxer du SN sujet. On observe le même phénomène en (14) dans le titre de la toile où le cuirassé The Warrior, ayant cessé son activité guerrière au sein de la Royal Navy, est représenté au repos (2.3).

15. Ainsi Serbat décrit-il le présent de l'indicatif comme «non-temporel » (Serbat, 1988: 20). Outre cet article de Serbat daté de 1988, Bosredon cite aussi «Le présent de l'indicatif et la catégorie du temps ", in Cl. Moussy et S. Mellet (éds.), 1992, La validité des catégories attachées au verbe. Lingua latina, Recherches linguistiques du Centre Alfred-Ernout, Paris, Presses Universitaires de Paris-Sorbonne, pp. 11-19.

16. L'on pense bien entendu à la généricité phrastique telle qu'elle est illustrée dans des exemples classiques comme The earth revolves around the sun ou Cats eat meat, qui comportent la forme non marquée du présent simple (cf. aussi 2.2 supra).

17. Le cas où le titre n'est pas une phrase (cf. note 6) est illustré par les syntagmes sans verbe ou avec un verbe de forme non finie tels que (2) Jacqueline les bras croisés, The Leaping Horse ou encore Le semeur.

18. L'existence de l'œuvre d'art était le cas que l'on rencontrait avec les traductions He greets / He salutes évoquées en note 11. On rapprochera également cette remarque de ce que l'on avait avec les exemples They did talk... / She slept... proposés plus haut en note 3. Si le prétérit exclut bien, en l'absence de toute mention d'une œuvre d'art, l'interprétation de ces phrases comme titres, on remarque également que, dans ce cas-là, le pronom de $3^{\mathrm{e}}$ personne du singulier she est anaphorique d'un SN dont le référent est occurrentiel.

19. Voir en particulier sur le sens d'adjectifs tels que adequate ou just, lié à l'appréciation et à la nécessité.

20. Cette coprésence peut même n'être qu'imaginée comme on l'a vu avec The Sleeping Negro en (15), qui est le titre d'un tableau en projet et non encore réalisé.

21. Le titre de l'ouvrage The Occupational Culture of the Boxer est traduit en français par La culture professionnelle du boxeur.

22. L'on aura noté que les développements ci-dessus réduisent la subjectivité et l'activité de représentation à celle du seul personnage. C'est volontairement que je n'ai retenu dans tout cet article que ce qui était strictement nécessaire à mon propos. Le lecteur pourra, s'il le souhaite, étendre l'analyse proposée ici d'un plan de référence représentationnel non «ordinairement déictique » en prenant en compte le discours du narrateur.

23. Pour un bref rappel de divers traitements du titre, voir Rebeyrolle, Jacques et Péry-Woodley (2009: 270).

24. C'est la raison pour laquelle ce titre était donné plus haut comme étant à bien des égards exemplaire (3.1.), dans lequel le présent simple fonctionne comme un signe de reconnaissance fort du caractère intitulatif de la phrase. S'agissant de la canonicité, il n'est que de penser à des titres moins canoniques comme D'où venons-nous? Que sommes-nous? Où allons-nous? (Paul 
Gaughin, 1897-1898), Take the Fair Face of Woman, and Gently Suspending, With Butterflies, Flowers, and Jewels Attending, Thus Your Fairy is Made of Most Beautiful Things (Sophie Gengembre Anderson, 1869 ; titre souvent abrégé en Take the Fair Face of Woman), You have no power over me et Birds are singing (Aurora Weinhold, contemporain) ou encore My Dad went to Howaii (Jenny Liang, 2015).

25. L'on se souvient que Langston Hughes (2.4), dans un contexte où la formule The Sleeping Negro était explicitement posée comme un titre de tableau, fournissait dans le contexte immédiatement à droite un commentaire de ce titre de tableau qui confirmait les analyses de Bosredon (1997) concernant la prototypie.

26. C'est aussi son statut de phrase avec verbe fini et ses connotations aspectuo-temporelles (cf. 2.4 et note 13) qui permet de l'articuler avec le récit, et une séquence telle que The sleeping boxer ne se serait pas insérée d'une manière aussi heureuse dans la narration. Je souhaite remercier ici Christiane Migette qui a bien voulu relire une dernière version de ce texte ; les erreurs ou autres insuffisances sont bien entendu les miennes.

\section{RÉSUMÉS}

Cet article analyse l'expression de la généricité par un syntagme nominal défini singulier comportant un nom discret au sein de la phrase The boxer sleeps, extraite d'un texte de fiction de Tennessee Williams. Cette phrase est interprétée comme un titre de tableau dans lequel le verbe au présent simple et le syntagme nominal sujet expriment tous deux une forme de généricité. Le syntagme nominal est vu comme un syntagme générique intitulatif de l'œuvre d'art visuelle, qui renvoie à un type (vs occurrence) bien particulier puisqu'il ne peut se passer de la coprésence de l'occurrence que constitue l'œuvre d'art. Tant la phrase étudiée que le contexte fournissent les moyens linguistiques d'une interprétation correcte de la phrase comme titre de tableau et de son syntagme

This article analyses the generic interpretation of a singular definite noun phrase containing a discrete noun within the sentence The boxer sleeps, which is to be found in a piece of literary fiction by Tennessee Williams. This sentence is construed as a painting title in which the simple present form of the verb and the subject noun phrase both express some kind of generic meaning. The noun phrase is seen as a titling generic phrase typical of visual works of art, which refers to a type (vs. occurrence) which can only exist together with an occurrential work of art. Both sentence and context provide the linguistic clues for the correct interpretation of the sentence as a painting title and that of its subject noun phrase as a titling generic noun phrase typical of visual works of art.

\section{INDEX}

Mots-clés : sémantique, syntagme nominal singulier défini, présent simple, titres de tableaux, type et occurrences, généricité, analyse de discours, fiction littéraire 


\section{AUTEUR}

\section{VIVIANE ARIGNE}

Université Paris13-Sorbonne-Paris Cité, Pléiade EA 7338viviane.arigne@orange.fr 